\title{
Numerical Modelling of Dynamic Resistance in a Parallel-Connected Stack of HTS Coated-Conductor Tapes
}

\author{
Justin M. Brooks, Mark D. Ainslie, Senior Member, IEEE, Zhenan Jiang, Senior Member, IEEE, \\ Stuart C. Wimbush, Rodney A. Badcock, Senior Member, IEEE, and Chris W. Bumby
}

\begin{abstract}
Dynamic resistance is observed in type-II superconductors carrying a DC transport current while simultaneously exposed to an alternating magnetic field. The appearance of a nonzero resistance is attributed to the interaction between the transport current and moving fluxons. This effect is relevant to many superconductor applications such as high-temperature-superconductor (HTS) flux pumps, DC / AC magnets, synchronous machines, and persistent current switches. Here, we present a finite element method (FEM) analysis of both the time averaged dynamic resistance and the instantaneous current sharing behaviour in a cable comprised of a stack of four YBCO thin films connected in parallel. Numerical modelling was performed using the $\boldsymbol{H}$-formulation method implemented in the commercial software COMSOL. The model employs experimentally measured values of the angular dependence of the critical current $I_{\mathrm{c}}(B, \theta)$ and the flux creep exponent $n(B, \theta)$. A single threshold field is observed, above which a finite dynamic resistance is observed in all tapes simultaneously. The time-averaged dynamic resistance of individual tapes tends to be larger for the exterior tapes than the interior tapes, but this difference decreases as the total transport current in the cable increases. We attribute this to shielding currents flowing in the exterior tapes during the majority of the cycle, which displace net DC current into the interior tapes. However, the relative proportion of DC transport current flowing in the exterior and interior tapes is also observed to vary periodically once per half cycle of the applied field. This is due to the periodic trapping of return screening currents in the interior tapes.
\end{abstract}

Index Terms-Dynamic resistance, $\boldsymbol{H}$-formulation, HTS modelling, HTS Cable, Parallel Stack

\section{INTRODUCTION}

$\mathbf{T}$ HE commercial availability of $\mathrm{km}+$ lengths of HTS coated conductor allows for the production of HTS devices such as HTS transformers, DC and AC magnets, flux pumps [1-5], motors/generators and persistent current switches $[6,7]$. In some of these applications, a coated conductor carrying a DC transport current is exposed to an oscillating magnetic field. These conditions are known to cause a non-zero resistance to

Manuscript receipt and acceptance dates will be inserted here. Financial support for this work was provided by New Zealand MBIE Endeavour grant no. RTVU1707 and NZ Royal Society Marsden Grant no. MFP-VUW1806. (Corresponding author: J. M. Brooks.)

J.M. Brooks, Zhenan Jiang, S.C. Wimbush, R.A Badcock and C.W. Bumby are with the Robinson Research Institute, Victoria University of Wellington, Lower Hutt 5046, New Zealand (e-mail: gus.brooks. @ vuw.ac.nz).

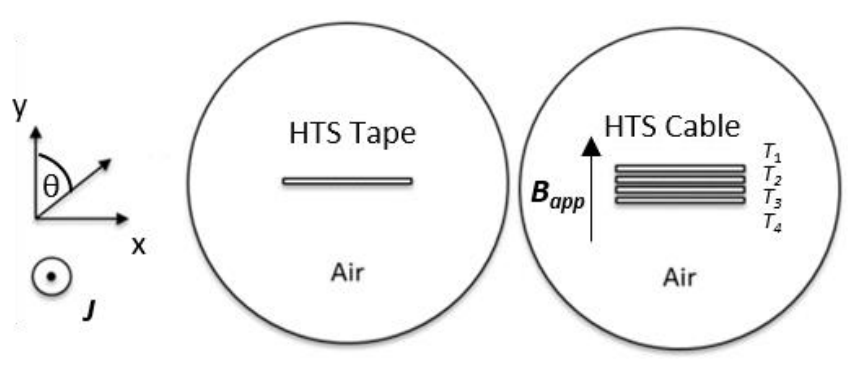

Fig. 1. FEM model architecture detailing the orientation of coordinate axes, and direction of transport current, $\boldsymbol{J}$, (into the page) and applied magnetic field, $\boldsymbol{B}_{\text {app }}$, relative to both the single wire and parallel-connected stacks.

arise in a superconducting material, due to dissipative interactions occurring between the transport current and moving fluxons [8-11]. This effect is termed the dynamic resistance. The dynamic resistance is often treated as a DC effect [12-16], but the time dependence of the voltage waveform along single tapes has also been measured [17-19] and simulated [20]. A clear understanding of dynamic resistance in HTS tapes and cables is important as the associated losses with this effect must be absorbed by the cryogenics of the system to avoid heating and possible damage to the HTS components. To date, most work on dynamic resistance has focused on single tapes, where analytical and finite-element (FEM) models have been shown to deliver close agreement with experiment. However, there has been little work on stacks of parallel-connected tapes such as occurs within high current cables. In such stacks, interacting self-fields from adjacent stacks in the tape can be expected to play a significant role - and experiments on series-connected stacks [21,22] diverge substantially from the expected analytical behaviour. In this work, we probe the dynamics and current sharing at play in a parallel-connected stack of tapes under dynamic resistance conditions, by utilizing an FEM model that has

M. D. Ainslie is with the Bulk Superconductivity Group, Department of Engineering, University of Cambridge, Trumpington Street, Cambridge CB2 1PZ, United Kingdom

Color versions of one or more of the figures in this paper are available online at http://ieeexplore.ieee.org.

Digital Object Identifier will be inserted here upon acceptance. 
been previously validated against experiments on a single tape $[20,23]$. Our results illustrate that naïve application of existing analytical solutions do not describe this situation well, and that current sharing between the tapes in the stack varies significantly over the AC field cycle. It is important to note that experimental 'short-sample' measurements of this type of parallel-stack arrangement are not practically possible, (due to the difficulty in obtaining perfect current sharing at parallel soldered joints). Therefore, FEM modelling represents the only feasible approach to undertake this investigation.

Exact analytical solutions can be obtained for the magnetic field and current density within a single superconducting strip supporting a DC transport current while exposed to a perpendicular oscillating magnetic field [24-27]. These solutions are used in $[8,28]$ to explain the underlying mechanism for the dynamic loss. A superconducting strip carrying a transport current distributes the current primarily at the surface of the material (ie the edge for thin films) so that the bulk of the material is shielded from a change in magnetic field. When an alternating magnetic field is superimposed, screening currents occupy the edge regions and the DC transport current is driven towards the center. If the amplitude of the oscillating magnetic field is less than some sample dependent threshold field $B_{\text {th }}$, the screening currents shield the interior regions from a change in magnetic flux. As a result, any transport currents flowing in this central region flow with no resistance. However, once the amplitude of the external field exceeds $B_{\mathrm{th}}$, the entire conductor experiences a change in flux density during the applied field cycle. In this case a non-zero dynamic resistance is observed, due to work done by the power supply in applying a Lorentz force to flux that traverses the transport current carrying region of the film.

Both $[8,28]$ state that, for a superconducting thin film of width $2 w$ and thickness $d$, critical current $I_{\mathrm{c} 0}$, in an alternating perpendicular field of amplitude $B_{\mathrm{a} 0}$ and frequency $f$, the time-averaged dynamic resistance $R_{\mathrm{dyn}}$ is given by:

$$
\frac{R_{d y n}}{f L}=\frac{4 w}{I_{c 0}}\left(B_{\mathrm{a} 0}-B_{t h}\right)
$$

However, these two papers give differing expressions for the threshold field $B_{\text {th }}$ as a function of the reduced current $i=I_{\mathrm{DC}} /$ $I_{\mathrm{c} 0}$ :

$$
\begin{aligned}
& B_{t h}=\frac{4.9284 \mu_{0} J_{c 0} d}{2 \pi}(1-i) \\
& B_{t h}=\frac{\mu_{0} J_{c 0} d}{2 \pi}\left[\frac{1}{i} \ln \left(\frac{1+i}{1-i}\right)+\ln \left(\frac{1-i^{2}}{4 i^{2}}\right)\right]
\end{aligned}
$$

Equation (2) is obtained from [8], whilst (3) is from [28].These expressions yield broadly similar results when the reduced current $i \gtrsim 0.1$ but diverge once $i<0.1$. In this low current region equation (3) delivers better agreement with experiments on single tapes [16]. The geometry of a stack of tapes is significantly different to that for which equations (1), (2), and (3) are derived, although the stack still exhibits a high-aspect ratio conductor cross-section. It is therefore not readily apparent whether these equations remain a valid predictor of the behaviour of the parallel stack. In order to investigate this, an FEM model has been

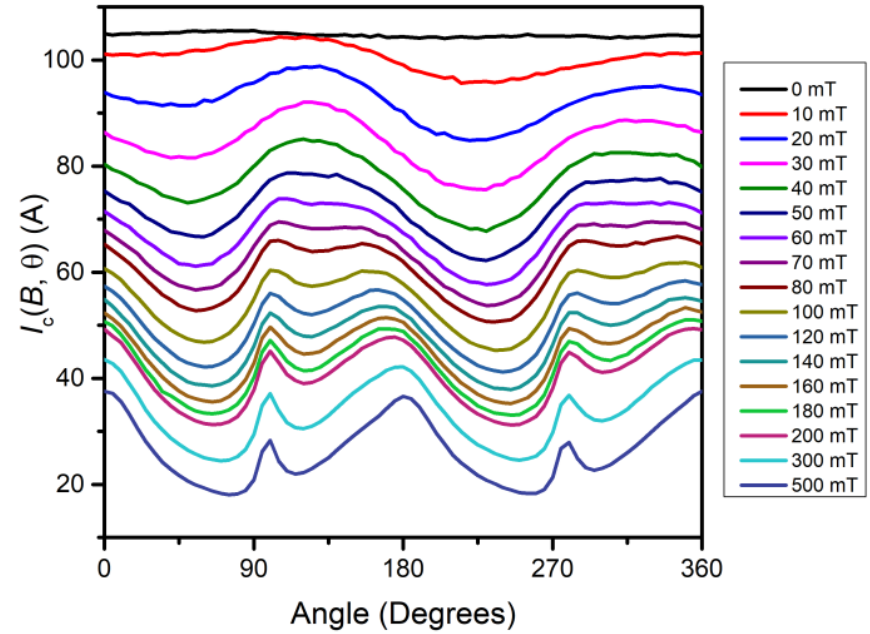

Fig. 2. Experimentally measured $I_{\mathrm{c}}(\boldsymbol{B}, \theta)$ data obtained at $77 \mathrm{~K}$ for the SuperPower studied in this work. This data was used as an input for the FEM models.

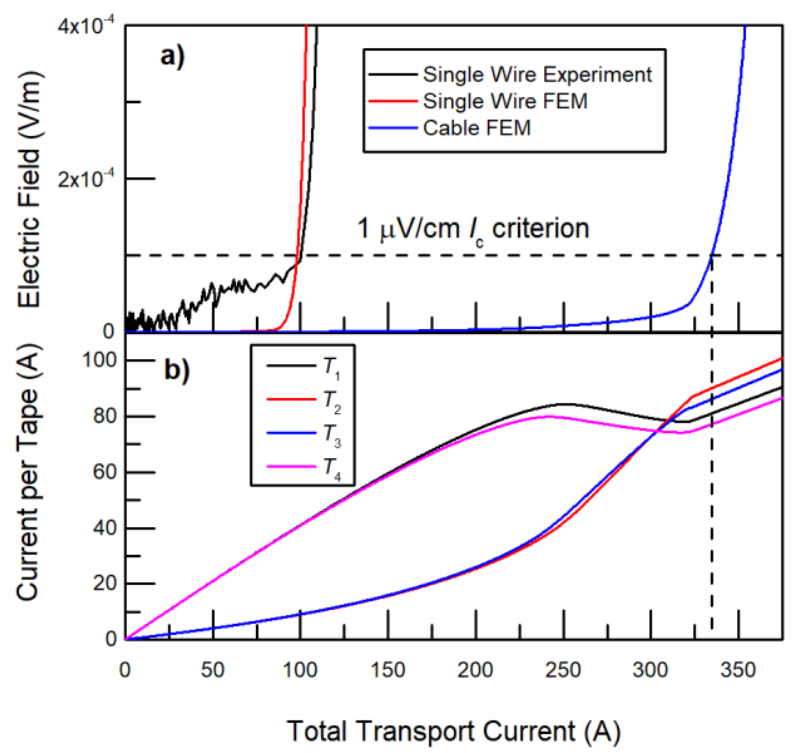

Fig. 3. (a) $I-V$ curves for a ingle tape as measured experimentally in self-field and simulated using our FEM model. This is shown alongside the simulated $I$ - $V$ curve for the cable in self-field. The horizontal dashed line shows $I_{\mathrm{c}}$ as defined by the $1 \mu \mathrm{V} / \mathrm{cm}$ criterion. (b) DC current within each tape of the cable during the DC ramp. The vertical dashed line indicates the critical current according to the $1 \mu \mathrm{V} / \mathrm{cm}$ criterion.

used to numerically calculate the local current density, field and dynamic resistance values within the cable stack and compare with the analytical expressions.

\section{Modelling Methodology}

Numerical calculations of the dynamic resistance were computed using the finite element method (FEM) commercial software COMSOL. The model architecture is shown in Fig. 1. for both a single coated conductor tape, and a cable comprising a 
stack of four tapes. We employed a $2 \mathrm{D} \boldsymbol{H}$-formulation model in the xy-plane (assuming an infinitely long tape in the z-direction). Numerical solutions were obtained through solving a combination of Faraday's law and Ampere's law.

$$
\begin{aligned}
& \boldsymbol{\nabla} \times \boldsymbol{E}=-\mu_{\mathbf{0}} \frac{\partial\left(\mu_{\mathrm{r}} \boldsymbol{H}\right)}{\partial t} \\
& \boldsymbol{\nabla} \times \boldsymbol{H}=\boldsymbol{J}
\end{aligned}
$$

Here $\boldsymbol{H}=\left[H_{x}, H_{y}\right]$ represents the magnetic field strength, $\boldsymbol{J}=$ $\left[J_{z}\right]$ is the current density and $\boldsymbol{E}=\left[E_{\mathrm{z}}\right]$. The constitutive relationship between $\boldsymbol{E}$ and $\boldsymbol{J}$ for the HTS cross-section is governed by an $\boldsymbol{E}$ - $\boldsymbol{J}$ power law $[29,30]$ of the form

$$
\boldsymbol{E}=\frac{E_{\mathbf{0}}}{J_{\boldsymbol{c}}(B, \theta)}\left|\frac{J}{J_{\boldsymbol{c}}(B, \theta)}\right|^{n(B, \theta)-1} \boldsymbol{J}
$$

The resistivity of the superconductor is thus the coefficient of $J . E_{0}$ is the standard electric field criterion of $1 \mu V / \mathrm{cm} . J_{\mathrm{c}}(B, \theta)$ and $n(B, \theta)$ are the critical current density and flux creep exponents. These are a function of the local magnetic field magnitude, $B$, and orientation, $\theta$, as shown in Fig. 2. Values for these two variables were obtained experimentally from measurements on a SuperPower SCS4050- AP tape using the 'SuperCurrent' measurement system at Robinson Institute [31, 32 ] at $77 \mathrm{~K}$. Measured values were corrected for self-field effects using the technique given in [33] and incorporated into the model using a two variable interpolation function $[34,35]$.

The current and field within the tape are computed in two stages during the model. Solutions were stored every $1 \mathrm{~ms}$ and $0.01 \mathrm{~ms}$ for the two stages. First, a DC transport current, $I_{\mathrm{DC}}$, is introduced under zero applied field and is ramped at a constant rate of $100 \mathrm{~A} / \mathrm{s}$ from zero until the desired transport current is reached. In the case of the four-tape stacked cable, the current is injected using the constraint $I_{\mathrm{DC}}=I_{\mathrm{T} 1}+I_{\mathrm{T} 2}+I_{\mathrm{T} 3}+I_{\mathrm{T} 4}$ to represent an ideal parallel connection between the four tapes in the stack. Here $I_{T 1}, I_{T 2}, I_{T 3}$, and $I_{T 4}$ represent the total DC current contained in the cross-sections of each tape in the cable. Once the desired current is reached, a sinusoidal perpendicular field of the form $B_{\text {app }}=B_{\mathrm{a} 0} \sin (\omega t)$ is then applied to the magnetic field boundary condition on the outer edge of the surrounding circular air sub-domain. $B_{\mathrm{a} 0}$ is increased in $5 \mathrm{mT}$ increments from 0 up to $100 \mathrm{mT}$ and the applied field frequency is $65 \mathrm{~Hz}$. These oscillations are run for two and a half cycles to ensure periodicity in the calculated current and field distributions. In the following analysis, we neglect the first cycle of the applied field where the films are magnetized from their virgin state.

Time-averaged values for the DC dynamic resistance are computed for each individual tape in the stack over the full cycle of the applied field, as follows. First the spatially averaged electric field $E_{\mathrm{Tn}}$ is determined over the cross-section $A_{\mathrm{Tn}}$ of a given tape.

$$
E_{\mathrm{Tn}}(t)=\frac{1}{A_{\mathrm{Tn}}} \iint_{A_{\mathrm{Tn}}} E_{\mathrm{Tn}}(x, y, t) d x d y
$$

a) $140 \mathrm{~A}$

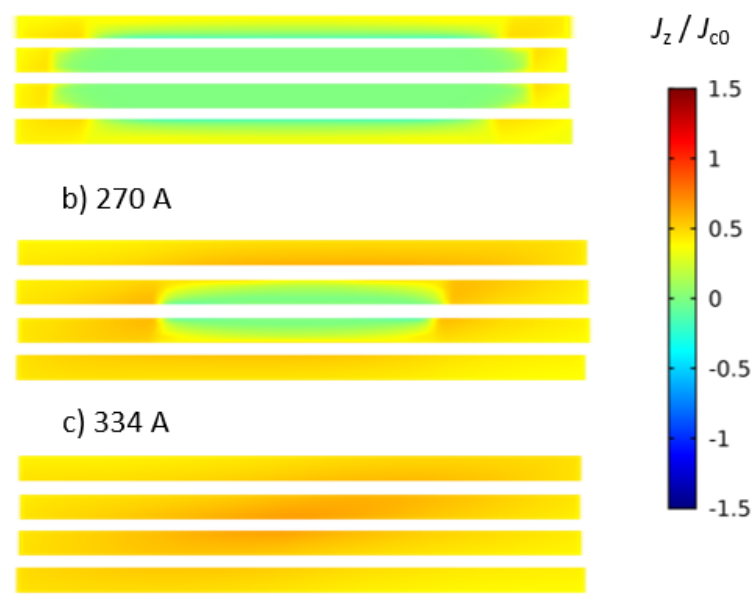

Fig. 4. Model cross-sections displaying the current density as a function of position at different times during the DC ramp. Plots (a), (b) and (c) show $J_{\mathrm{z}} / J_{\mathrm{c} 0}$ at $140 \mathrm{~A}, 270 \mathrm{~A}$ and $334 \mathrm{~A}$. The tape aspect ratio has been adjusted to assist the viewer.

where $T n$ denotes the position of the tape in the stack (ie $T_{1}$, $T_{2}, T_{3}$ or $T_{4}$ ). The spatially-averaged electric field is then divided by the DC current, $I_{\mathrm{Tn}}$, contained within that cross-section to produce the time-averaged dynamic resistance for a given tape over a single cycle of the applied field

$$
\frac{R_{\mathrm{dyn}, \mathrm{T} n}}{f L}=\int_{0}^{\frac{1}{f}} \frac{E_{\mathrm{Tn}}(t)}{I_{\mathrm{T} n}(t)} d t
$$

The dynamic resistance of the full parallel connected stack is then obtained from:

$$
\frac{1}{R_{d y n, \text { cable }}}=\sum_{n=1, \ldots 4} \frac{1}{R_{d y n, T n}}
$$

We assume that losses occur only in the HTS material, thus the model consists of the HTS layer of each tape which is $4 \mathrm{~mm}$ wide and $1 \mu \mathrm{m}$ thick, each separated in the y-direction by a centre-to-centre distance of $100 \mu \mathrm{m}$. The entire stack is then surrounded by a circular air domain, as shown in Figure A.1 (see Appendix). The HTS layer meshing is a uniform grid consisting of 100 elements along the width of the conductor ( $x$-axis) and 3 elements over its thickness ( $y$-axis). This approach minimizes the computational time for the simulation whilst retaining resolution at the surface of the HTS domain. A free triangular mesh (determined by COMSOL) is used in the surrounding air subdomain. The spatial resolution of this free mesh increases in the vicinity of each HTS layer, in order to match nodes at the layer boundary. The air domain boundary has a radius of $4 \mathrm{~cm}$ and is sufficiently far away from the stack that the normal component of the magnetic field due to the superconductor is negligible at the boundary, and is hence set to zero. In the air sub-domain, we solve Ohms law with the resistivity set to $1 \Omega \mathrm{m}$. 


\section{Modelling Results}

\section{A. I-V curves and current sharing in zero applied field}

Fig. 4(a) shows the numerically calculated $I$ - $V$ curves obtained when ramping the DC current in both a single tape and the cable under zero applied field (ie self-field). Experimental data obtained from a self-field transport measurement on a short sample of the SuperPower SCS4050-AP YBCO tape [36] is also shown. The experimental $I_{\mathrm{c} 0}$ of the Superpower tape studied in this work is $105.6 \mathrm{~A}$ and the power law exponent $n$ is 27. $J_{\mathrm{c} 0}$ is determined by dividing $I_{\mathrm{c} 0}$ by the area of the HTS film cross-section. A small non-zero voltage is experimentally measured for the single tape at currents below $I_{\mathrm{c}}$, which we attribute to a combination of instrumental drift and non-ideal tape behavior. Nonetheless, at currents approaching and above $I_{\mathrm{c}}$, experimental results and model agree well.

It is not generally possible to make experimental measurements on a short sample (e.g. less than several meters) of a parallel stack of tapes, as small variations in the soldered contact resistance to each tape significantly affect current sharing between the tapes [37]. Instead we must rely on numerical modelling to determine behavior in this arrangement. The experimentally measured and FEM model results for the single tape $I_{\mathrm{c} 0}$ are in close agreement at $100.5 \mathrm{~A}$ and $102.6 \mathrm{~A}$, respectively. The stacked cable FEM model gives a cable critical

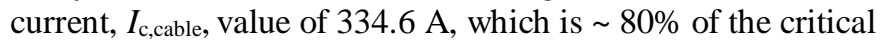
current of four isolated coated conductor tapes. We attribute this relative reduction in $I_{\mathrm{c} \text { cable }}$ to the increased self-field due to the other tapes in close proximity within the stack. This has also been observed in a series-connected stack of tapes reported in [21].

As DC current is injected into the cable, it must distribute itself amongst the four tapes. This is shown in Fig. 3(b), where we observe that at first current is injected primarily into the outer tapes in the stack $\left(T_{1}\right.$ and $\left.T_{4}\right)$. As the total current in the cable increases, the share of the total current carried by the inner tapes $\left(T_{2}\right.$ and $\left.T_{3}\right)$ increases. Once $I_{\mathrm{DC}}$ reaches approximately

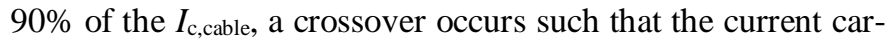
ried within the inner tapes then exceeds the current carried by the outer tapes. Above $I_{\mathrm{c} \text {,cable }}$ any additional transport current is then distributed evenly between the four tapes - as can be seen by the equal linear gradients for each tape beyond this point.

Further understanding of the evolving current distribution in the stack during the initial current injection can be obtained by examining the current density across the full cross section of the stacked cable. Figs. 4 (a), (b), and (c) show the current density as a fraction of $J_{\mathrm{c} 0}$ at three moments during the current ramp; when $I_{\mathrm{DC}}$ is $140 \mathrm{~A}, 270 \mathrm{~A}$, and $334.6 \mathrm{~A}$. The parallel connected stack can be considered to behave similarly to a superconducting bulk, in the sense that when $I_{\mathrm{DC}}$ is well below $I_{\mathrm{c} \text {,cable, the }}$ current density is largest near the surface. This manifests as current flowing largely across the entire width of the outermost tapes, $T_{1}$ and $T_{4}$, as well as at both edges of the interior tapes, $T_{2}$ and $T_{3}$. As the total transport current is increased, the outer tapes become saturated with DC transport current across their entire cross section, leaving a small shielded region which exists only in the central regions of tapes $T_{2}$ and $T_{3}$. At $I_{\mathrm{c} \text {,cable }}$ this shielded

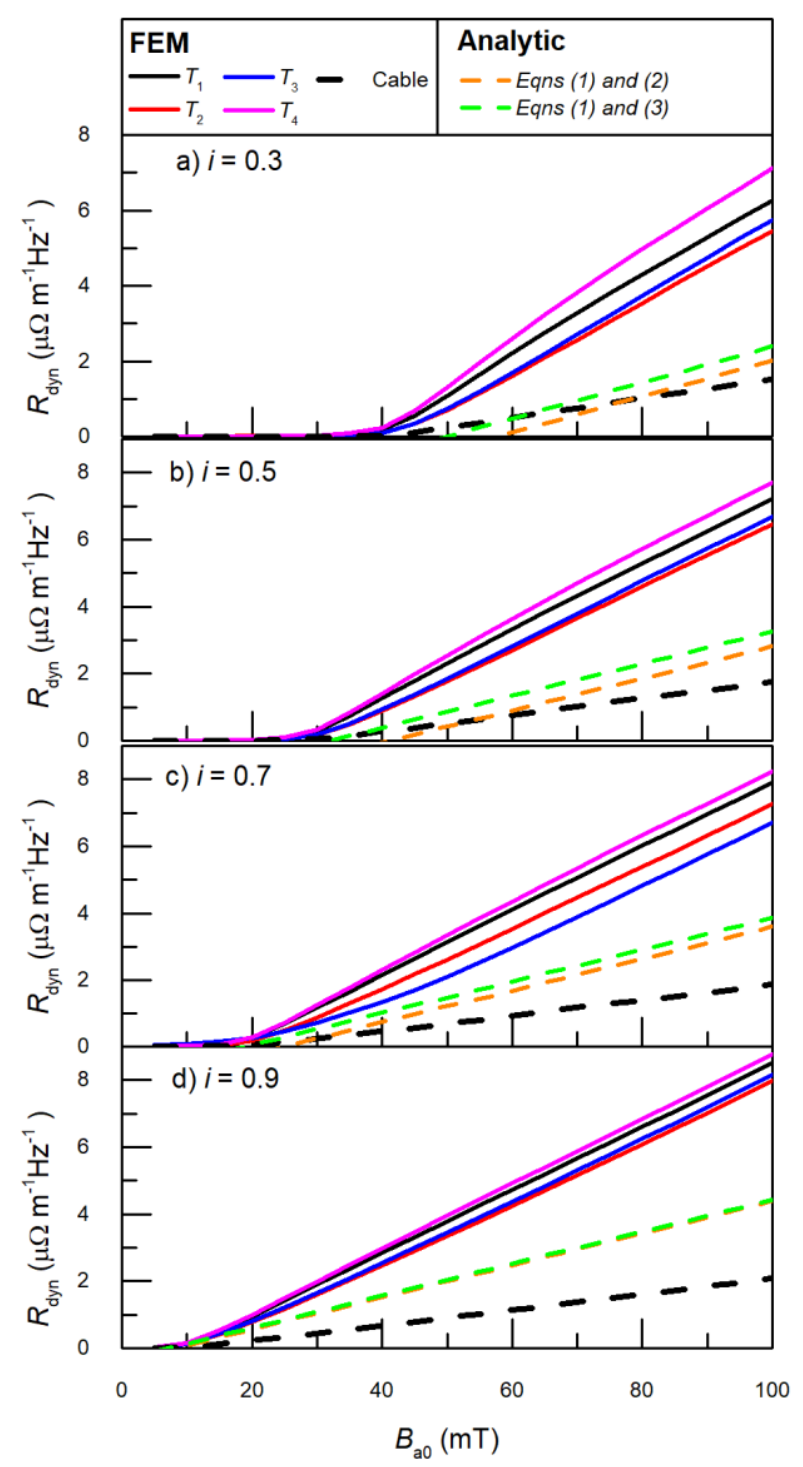

Fig. 5. Time averaged values for the dynamic resistance and threshold fields. Plots (a), (b), (c), and (d) show the time averaged resistance for four different values of $i=0.3,0.5,0.7$, and 0.9 in AC field amplitudes up to $100 \mathrm{mT}$.

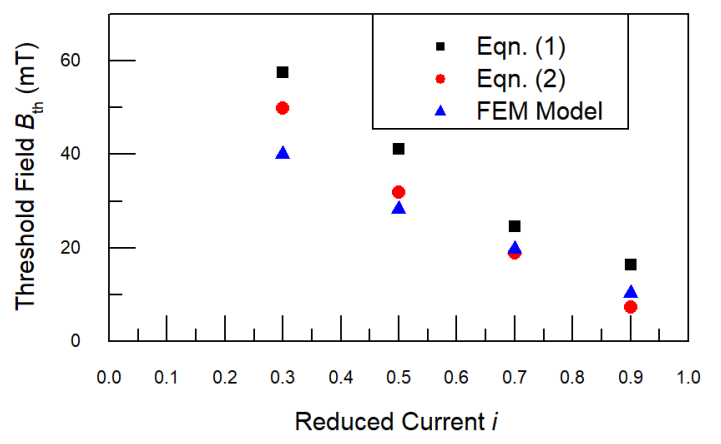

Fig. 5. $B_{\text {th }}$ values calculated using from the FEM model and equations (2), and (3). 
region disappears, and each tape carries a critical current determined by its $J_{\mathrm{c}}(B, \theta)$ properties and the local magnetic environment. The inner tapes experience a smaller field contribution from their neighbouring tapes than the outer tapes, which is why the inner tapes carry a slightly larger proportion of the total current. The reason for this is that the inner tapes have neighbouring tapes both above and below and this symmetry leads to the normal contribution from each neighbor being partially cancelled out. By contrast the outer tapes experience the full contribution from their single neighbouring tape, and hence experience a larger perpendicular field at $I_{\mathrm{c}, \text { cable }}$.

\section{B. DC Values for the dynamic resistance and threshold field}

Figs. 5(a)-(d) show the values calculated from eqn. 8 for the DC dynamic resistance, $R_{\mathrm{dyn}}$ per cycle, for each tape and the cable using the FEM model. Analytical values for the cable obtained from equations (1), (2), and (3) are also shown. $R_{\text {dyn }}$ is calculated for four different values of the reduced current $i=$ $0.3,0.5,0.7,0.9$, and for a single frequency $f=65 \mathrm{~Hz}$. The $R_{\mathrm{dyn}}$ values are plotted as a function of the applied field amplitude $B_{\mathrm{a} 0}$.

For all reduced currents, there is a single threshold field value below which the DC values for the dynamic resistance in each tape is negligible. Only once the applied field amplitude exceeds this threshold value, does the time-averaged resistance for each tape begin to linearly increase. This is because the parallel connection of tapes in the stack ensures that trans port current is dynamically redistributed such that the potential drop along each tape is identical. This situation differs

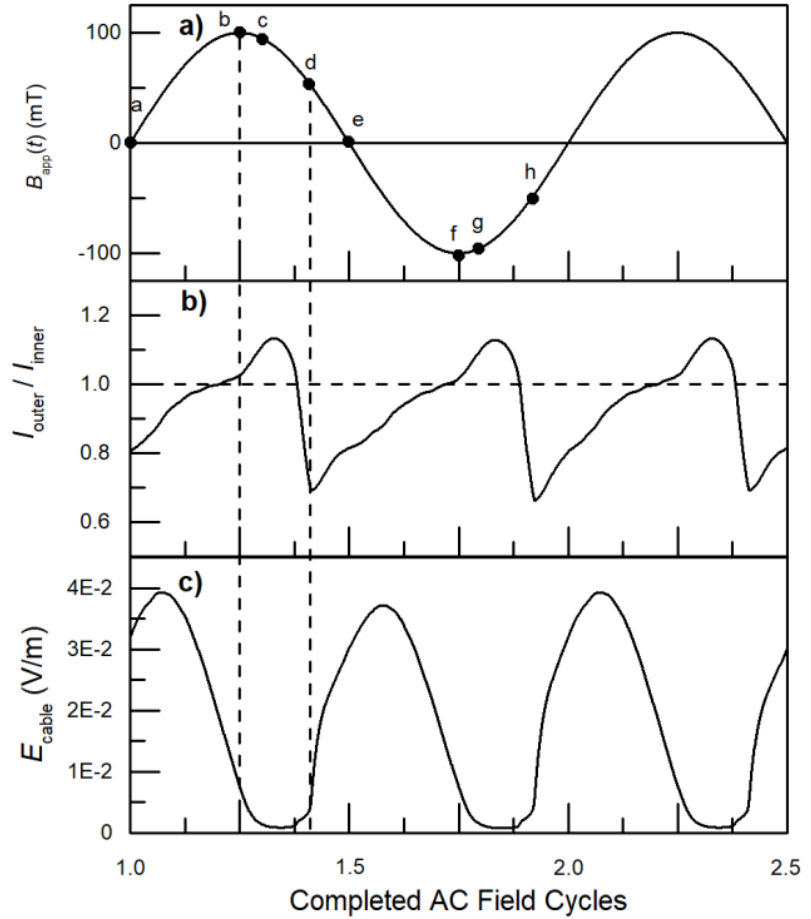

Fig. 7. (a) Phase of the applied magnetic field with $B_{\mathrm{a} 0}=100 \mathrm{mT}$. (b) and (c) show the time dependence of $I_{\text {outer }} / I_{\text {inner }}$ and $E_{\text {cable }}$ for $i=0.5$. These plots span 1.5 cycles of the applied field. The solid black circles in (a) indicate the eight instances in time referenced in figure 8 . The dashed lines indicate when the applied magnetic field $B_{\mathrm{app}}=B_{\mathrm{a} 0}$ and $B_{\mathrm{a} 0}-2 B_{\mathrm{th}}\left(B_{\mathrm{th}}\right.$ calculated using eqn. 3$)$ a) Completed AC Field Cycles $=1, B_{\mathrm{a} 0}=0 \mathrm{mT}$

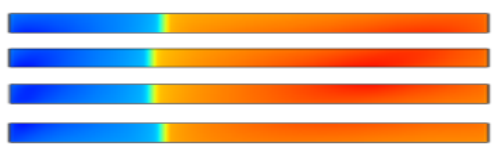

b) Completed AC Field Cycles $=1.25, B_{\mathrm{a} 0}=100 \mathrm{mT}$

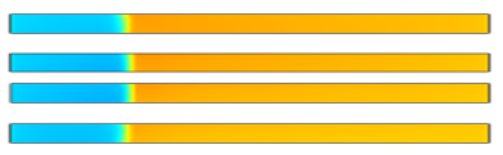

c) Completed AC Field Cycles $=1.333, B_{\mathrm{a} 0}=86 \mathrm{mT}$

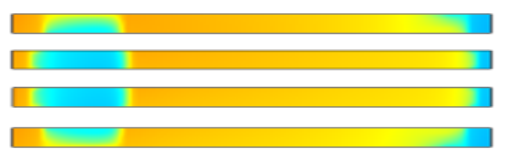

d) Completed AC Field Cycles $=1.41, B_{\mathrm{a} 0}=53 \mathrm{mT}$

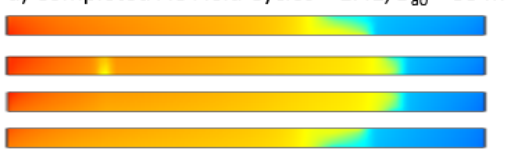

e) Completed AC Field Cycles $=1.5, B_{a 0}=0 \mathrm{mT}$

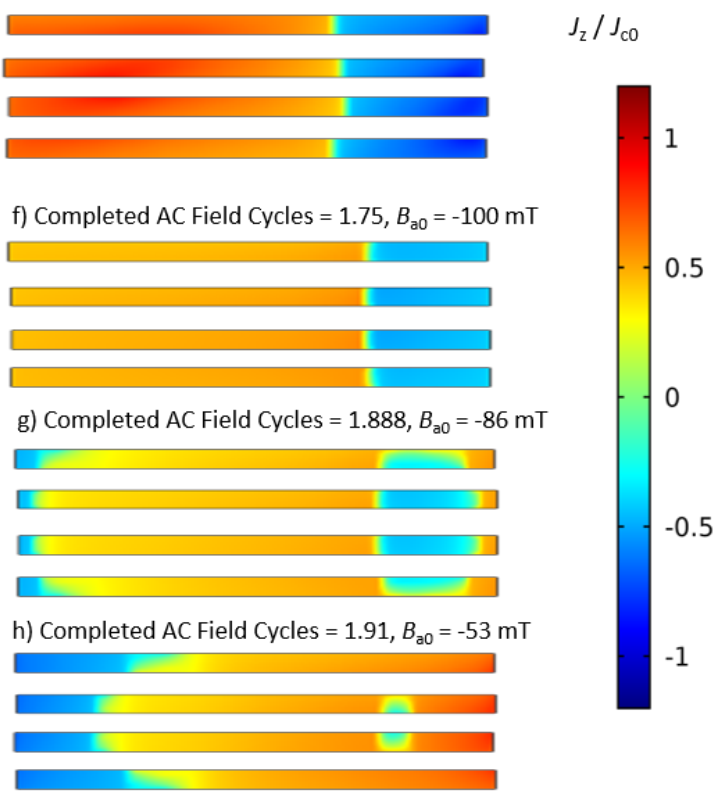

Fig. 8. Model cross-sections showing $J_{\mathrm{z}} / J_{\mathrm{c} 0}$ within the cable as a function of position at eight separate instances during a single cycle as indicated by the solid black circles (a-h) in Fig. 7(a). The magnitude of the current density is indicated by the color scale on the right-hand side. 
from a series-connected stack of tapes [21], where the transport current in each tape is constrained to be equal and hence shielding effects mean that dynamic resistance emerges in the outer tapes well before it is observed in the inner tapes.

For all values of $i$, the individual exterior tapes in the parallel stack have a larger DC dynamic resistance compared to the interior tapes. This is because they carry a greater proportion of shielding currents, and consequently a smaller proportion of the total transport current than the inner tapes. When $i=0.9$, the difference between the dynamic resistance of each tape decreased significantly. This reflects the fact that the cable can support less screening currents at highly elevated values of $i$.

Fig. 6 shows values for $B_{\text {th }}$ of the total stacked cable, calculated using (2), and (3). These are shown alongside values obtained from the FEM model. The FEM model values for $B_{\text {th }}$ were obtained from the $x$-intercept of linear fits to the nonzero section of $R_{\mathrm{dyn}}$ in each tape, which were then averaged. We obtain values of surprisingly similar magnitude from the analytical equations for a superconducting strip and the FEM results for the 4-tape stack, despite the different underlying geometry. This reflects that the 4-tape stack has a total height of only $\sim 300 \mu \mathrm{m}$ and a width of $4 \mathrm{~mm}$, so its aspect ratio remains large and can be approximated by treating as currents flowing in a single plane. It should be noted that larger cable stacks containing more tapes in parallel should not be expected to display the same close approximation to strip behaviour.

\section{Transient electric field and current sharing in a sinusoi- dal perpendicular field}

The DC values for $R_{\text {dyn }}$ presented in section III.B are calculated from the transient instantaneous values for the transport current density and average electric field in each tape in the cable. These transient quantities vary over the alternating field cycle. In particular, screening currents redistribute during the cycle which causes the net DC transport current component to also move between the exterior and interior tapes. This is illustrated in Fig. 7(b) which presents the time-dependence of the ratio of transport current carried by the outer and inner tapes respectively when $i=0.5$ and $B_{\mathrm{a} 0}=100 \mathrm{mT}$. This is expressed as $I_{\text {outer }} / I_{\text {inner }}=\left(I_{\mathrm{T} 1}+I_{\mathrm{T} 4}\right) /\left(I_{\mathrm{T} 2}+I_{\mathrm{T} 3}\right)$. Fig. 7 (c) shows how this varying ratio also corresponds to the transient electric field across the stack, $E_{\text {cable. }}$. Note that the ideal parallel connection between every tape in the stack means that $E_{\text {cable }}=E_{\mathrm{Tn}}$ for all tapes).

In Figs. 7(b) and (c), we see the evolution of these quantities over 1.5 cycles of the applied AC field.The dashed lines show the window in which the applied magnetic field decreases from its peak value of $B_{\mathrm{a} 0}$ to approximately $B_{\mathrm{a} 0}-2 B_{\mathrm{th}}$ (calculated using eqn. 3), This is the point in the cycle at which the Bean model predicts screening current reversal is no longer capable of shielding the interior from the changing external field [21], and hence holding the interior electric field at its minimum value. Immediately following this window, we then observe a sharp rise in $E_{\text {cable }}$ and simultaneously a greater proportion of the DC current moves into the interior tapes. This occurs as the screening currents are driven in the outer tapes to oppose the rapidly changing applied field and hence the DC current component is driven into the inner tapes. The ratio of

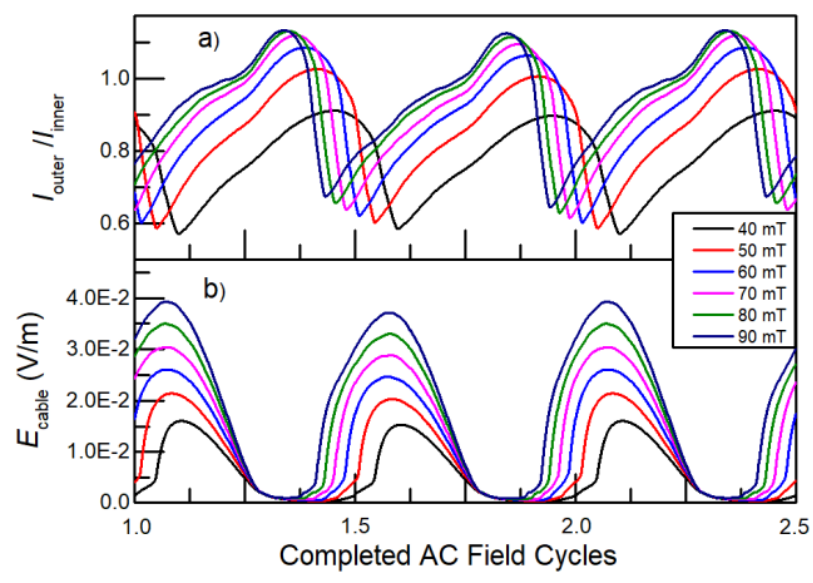

Fig. 9. Time dependence of (a) $I_{\text {outer }} / I_{\text {inner }}$ and (b) $E_{\text {cable }}$ for a range of $B_{\mathrm{a} 0}$ values over 1.5 cycles of the applied magnetic field for $i=0.5$.

DC current $I_{\text {outer }} / I_{\text {inner }}$, then steadily increases until the applied field reaches the opposite polarity peak and the process then repeats in the next half-cycle.

This narrative is presented visually in Fig. 8 with colour maps of $J_{\mathrm{Z}} / J_{\mathrm{c} 0}$ across the entire cable cross-section in the same fashion as Fig. 3. Here, eight separate moments during the AC applied field cycle are shown, which correspond to the numbered points in Fig. 7(a).

Fig. 8(a) shows the current density after a single full cycle of the applied magnetic field. At this moment $B_{\text {app }}=0 \mathrm{mT}$ and is increasing. Screening currents of opposite polarity to the DC current flow on the left-hand side of the cable and penetrate slightly further into the exterior tapes. The right-hand side of the cable accommodates the sum of screening currents and the DC transport current. At this instant in the cycle, dissipation is occurring across the entire conductor and $E_{\text {cable }}$ is increasing.

Fig. 8(b) shows the current distribution by $B_{\text {app }}=100 \mathrm{mT}$ after 1.25 cycles. At this moment $E_{\text {cable }}$ has fallen from its peak value and almost decayed to zero. The total induced screening currents flowing in the stack have decreased since Fig.8(a). This is evident from the reduced area of the return path on the left-hand side of the cable, along with the global reduction in the magnitude of the current density.

Fig. 8(c) shows the moment shortly after the applied field has reached its maximum, where $B_{\text {app }}=86 \mathrm{mT}$ and is now decreasing, . (This occurs after 1.333 complete cycles in Fig. 7(a)). Now, $E_{\text {cable }}$ is at its minimum value whilst $I_{\text {outer }}$ is just at its maximum. Interestingly, this is occurring sometime after $\mathrm{d} B_{\text {app }} / \mathrm{d} t=0$. Fig.8(c) shows new return paths for negative polarity screening currents appearing now on the opposite edge of the tape. At the same time, negative currents which were previously flowing on the left side are now being gradually erased by positive currents encroaching from the left edge. These residual screening currents vanish first in the exterior tapes leading to a short-lived peak in the DC current contained in the exterior tapes (see Fig 9(a)). Shortly afterwards the residual 'island' of screening currents is also erased from the interior tapes, which are then able to accommodate a greater proportion of the transport current. 
By Fig. 8(d), the applied field has dropped to $B_{\text {app }}=53 \mathrm{mT}$, and 1.41 AC field cycles have passed. At this moment, the negative screening currents on the left-hand side have vanished entirely. This coincides with the rapid increase from zero of $E_{\text {cable }}$ and a sudden decrease in $I_{\text {outer. }}$ It should be noted that this the applied field at this moment is approximately equal to $B_{\mathrm{a} 0}-2 B_{\mathrm{th}}$. After this moment current distributions which are the mirror image of those in fig. 8(a-d) are observed in fig.8 (e-h) as the AC field goes through the negative half of the AC field cycle.

The time-varying distribution of transport current between tapes is observed at all applied field amplitudes. Fig. 9 shows the time dependence of $I_{\text {outer }} / I_{\text {inner }}$ and $E_{\text {cable }}$ for a range of $B_{\mathrm{a} 0}$ values (with $B_{\mathrm{a} 0}>B_{\mathrm{th}}$ ). At increasing values of $B_{\mathrm{a} 0}$, the maximum value of $I_{\text {outer }} / I_{\text {inner }}$ increases, indicating that a greater proportion of the interior tapes are taken up by reversing currents during the period between $B_{\mathrm{a} 0}$ and $B_{\mathrm{a} 0}-2 B_{\text {th }}$ (equivalent to Fig. 8(c)). We also observe that the peaks in the $E_{\text {cable }}$ waveform broaden and increase in amplitude with increasing $B_{\text {ao }}$. This directly causes the DC value of $R_{\text {dyn }}$ to also increase as shown in Fig. 5.

\section{CONCLUSIONS}

We have modelled the dynamic resistance in a cable comprising a stack of four YBCO coated conductors connected in parallel. Our numerical model employed the $\boldsymbol{H}$-formulation and included the dependency of the critical current on the local magnetic flux density and orientation relative to the plane of the coated conductor film.

When the transport current flowing through the cable is held constant and an alternating field applied, we observe a single threshold field above which the time-averaged values for the dynamic resistance increases linearly from zero for all tapes. The FEM values for the time-averaged dynamic resistance and threshold fields have poor agreement with those from the analytical model (eqs.1-3). While the cable retains a high aspect ratio, it's behaviour cannot be described by single strip with a constant critical current density.

When we examine the transient behaviour of the cable electric field, we see that the transport current moves between flowing primarily in the exterior or interior tapes, depending on the phase of the applied magnetic field. When the rate of change of the applied field changes polarity, screening currents appear on the edges of the tape which act to erase the screening currents from the previous half cycle. During this period, the electric field goes through its minimum values and most of the transport current flows in the exterior tapes. After the screening currents are completely erased, the electric field rapidly increases due to a combination of the suppression of the critical current and the increasing presence of screening currents. In these moments, the DC transport flows mostly in the interior tapes.

For all values of the reduced current $i$, the calculated value for the time-averaged DC dynamic resistance exceeds 1 $\mu \mathrm{V} / \mathrm{cm}$ for samples with length in excess of $1 \mathrm{~m}$. Utilizing this as a switching mechanism in an HTS transformer rectifier is prohibited by the need for a large AC magnet bore to house such a long sample length. Other possible solutions include increasing the amplitude of the AC magnetic field, or winding the cable to make several passes through the bore of the exciting magnet.

Our results provide interesting insights into the complex current redistribution behavior that can be observed in nontransposed parallel connected cables of multiple ReBCO tapes. They also illustrate the utility of $\boldsymbol{H}$-formulation FEM models to study parallel connected cable arrangements which cannot easily be measured as short samples in the laboratory.

\section{ACKNOWLEDGEMENTS}

M.D. Ainslie acknowledges financial support from an EPSRC Early Career Fellowship EP/P020313/1. All data are provided in full in the results section of this paper.

\section{APPENDIX}

a)

b)
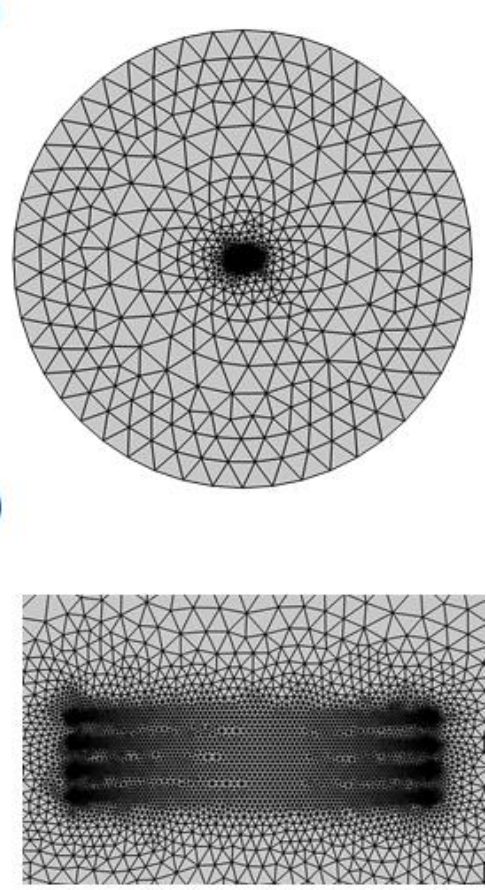

c)

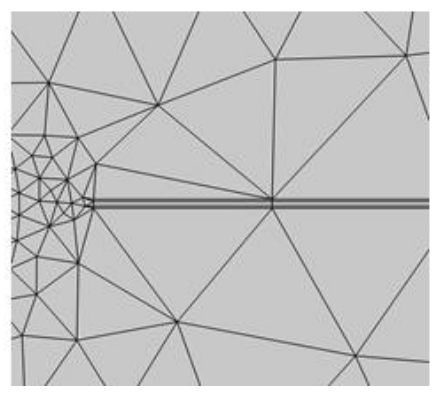

Fig. A1. Finite element meshing used for total COMSOL model (air + HTS). (a) Total domain is a 2D circle with radius $4 \mathrm{~cm}$, mesh resolution increases towards tape stack. (b) Magnified mesh in region of tapes (denoted by red rectangle in (a)), showing fine meshing around each tape. (c) 


\section{REFERENCES}

[1] J. Geng and T. Coombs, "Mechanism of a high-Tc superconducting flux pump: Using alternating magnetic field to trigger flux flow," Appl. Phys. Lett., vol. 107, no. 14, p. 142601, 2015.

[2] J. Geng, K. Matsuda, L. Fu, J.-F. Fagnard, H. Zhang, X. Zhang, B. Shen, Q Dong, M. Baghdadi, and T. Coombs, "Origin of dc voltage in type ii superconducting flux pumps: field, field rate of change, and current density dependence of resistivity," J. Phys. D, vol. 49, no. 11, p. 11LT01, 2016.

[3] Z. Jiang, K. Hamilton, N. Amemiya, R. Badcock, and C. Bumby, "Dynamic resistance of a high-Tc superconducting flux pump," Appl. Phys. Lett., vol. 105, no. 11, p. 112601, 2014.

[4] Z. Jiang, C. W. Bumby, R. A. Badcock, H.-J. Sung, N. J. Long, and N. Amemiya, "Impact of flux gap upon dynamic resistance of a rotating HTS flux pump," Supercond. Sci. Technol.,vol.28, no. 11, p. 115008, 2015.

[5] C. Bumby, Z. Jiang, J. Storey, A. Pantoja, and R. Badcock, "Anomalous open-circuit voltage from a high $\mathrm{T}_{\mathrm{c}}$ superconducting dynamo," Appl. Phys. Lett., vol. 108, no. 12, p. 122601, 2016

[6] C. Li, J. Geng, J. Gawith, B. Shen, X. Zhang, H. Zhang, J. Ma, and T. A. Coombs, "Design for a persistent current switch controlled by altern ating current magnetic field," IEEE Trans. Appl. Supercond., vol. 28, no. $4,2018$.

[7] J. Geng, K. Matsuda, B. Shen, H. Zhang, X. Zhang, L. Fu, Z. Huang, and T.Coombs, "HTS persistent current switch controlled by ac magnetic field," IEEE Trans. Appl. Supercond., vol. 26, no. 3, 2016.

[8] V. Andrianov, V.Zenkevich, V. Kurguzov, V. Sytchev, and F. Ternovskii, "Effective resistance of an imperfect type-II superconductor in an oscillating magnetic field." Sov. Phys._JETP., vol. 31 p. 815, 1970.

[9] M. Aikele, R. Huebener, D. Weischer, and C. Tsuei, "Effect of highfrequency magnetic fields on the dissipative vortex motion in the superconducting mixed state," Physica C, vol. 290, no. 1-2, pp. 109-112, 1997.

[10] M. Oomen, J. Rieger, M. Leghissa, B. ten Haken, and H. H. ten Kate, "Dynamic resistance in a slab-like superconductor with $\mathrm{I}_{\mathrm{c}}(\mathrm{B})$ dependence," Supercond. Sci. Technol., vol. 12, no. 6, p. 382, 1999.

[11] T. Ogasawara, K. Yasuköchi, and S. Sayama, "Effective resistance of current-carrying superconducting wire in oscillating magnetic fields 2 . twisted multifilamentary composite conductor," Cryogenics, vol. 16, no. 2, pp. 89-96, 1976.

[12] M. Ciszek, H. Knoopers, J. Rabbers, B. ten Haken, and H. H. ten Kate, "Angular dependence of the dynamic resistance and its relation to the ac transport current loss in bi-2223/ag tape superconductors," Supercond. Sci. Technol., vol. 15, no. 8, p. 1275, 2002.

[13] M. Ciszek, O. Tsukamoto, J. Ogawa, and D. Miyagi, "Energy losses in YBCO-123 coated conductors carrying transport current in perpendicular external magnetic field,"in AIPConf. Proc., vol.614,no.1., pp. 606-613, 2002

[14]Z. Jiang, R. Toyomoto, N. Amemiya, C. W. Bumby, R. A. Badcock, and N. J. Long, "Dynamic resistance measurements in a GdBCO-coated conductor," IEEE Trans. Appl. Supercond., vol. 27, no. 4, 2016.

[15] Z. Jiang, R. Toyomoto, N. Amemiya, X. Zhang, and C. W. Bumby, "Dynamic resistance of a high- $\mathrm{T}_{\mathrm{c}}$ coated conductor wire in a perpen- dicular magnetic field at 77 K," Supercond. Sci. Technol., vol. 30, no. 3, p. 03LT01, 2017.

[16] Z. Jiang, W. Zhou, Q. Li, M. Yao, J. Fang, N. Amemiya, and C. W. Bumby, "The dynamic resistance of ybco coated conductor wire: Effect of dc current magnitude and applied field orientation Supercond. Sci. Technol., vol. 31, no. 3, p. 035002, 2018.

[17] A. Uksusman, Y. Wolfus, A. Friedman, A. Shaulov, and Y. Yeshurun, "Voltage response of current carrying $\mathrm{Y}-\mathrm{Ba}-\mathrm{Cu}-\mathrm{O}$ tapes to alternating magnetic fields," J. Appl. Phys., vol. 105, no. 9, p. 093921, 2009.

[18] C. Li, J. Geng, J. Yang, J. Gawith, B. Shen, J. Ma, and T. Coombs, “Second harmonic in the voltage of dc-carrying YBCO tape under a perpendicular alternating magnetic field," Physica C, vol. 564, p. 11-16, 2019.

[19] R. C. Duckworth, Y.F. Zhang, T. Ha, and M. J. Gouge, "Dynamic resistance of YBCO-coated conductors in applied ac fields with dc transport currents and dc background fields," IEEE Trans. Appl. Supercond., vol. 21, no. 3, pp. 3251-3256, 2010.

[20] M. D. Ainslie, C. W. Bumby, Z. Jiang, R. Toyomoto, and N. Amemiya, "Numerical modelling of dynamic resistance in high-temperature superconducting coated-conductor wires," Supercond. Sci. Technol., vol. 31, no. 7, p. 074003,2018

[21] Z. Jiang, W. Zhou, C. Bumby, M. Staines, Q. Li, R. Badcock, N. Long, and J. Fang, "Dynamic resistance measurement of a four-tape YBCO stack in a perpendicular magnetic field," IEEE Trans. Appl. Supercond., vol. 28, no. 4, 2017.

[22] Y. Liu, Z. Jiang, Q. Li, C. W. Bumby, R. A. Badcock and J. Fang, "Dynamic Resistance Measurement in a Four-Tape YBCO Stack With Various Applied Field Orientation," IEEE Trans. Appl. Supercond. vol. 29, no. 5 , Art no. 4801507,2019

[23] Brooks, J.M, Ainslie, M.D, Jiang, Z., Pantoja, A.E, Badcock, R.A Bumby, C.W, "The Transient Voltage Response of REBCO Thin Films Exhibiting Dynamic Resistance.”, 2019, Submitted to Supercond. Sci. Technol.

[24] W. Norris, "Calculation of hysteresis losses in hard superconductors carrying ac: isolated conductors and edges of thin sheets," J. Phys. D, vol. 3, no. 4, p. $489,1970$.

[25] E. Brandt, M. Indenbom, and A. Fork1, "Type-II superconducting strip in perpendicular magnetic field,” Eur. Phys. Lett., vol. 22, no. 9, p. 735, 1993.

[26] E. H. Brandt and M. Indenbom, "Type-II-superconductor strip with current in a perpendicular magnetic field," Phys. Rev. B, vol. 48, no. 17, p. 12893, 1993.

[27] E.Zeldov, J. R. Clem, M. McElfresh, and M. Darwin, "Magnetization and transport currents in thin superconducting films," Phys. Rev. B, vol. 49, no. 14 , p. $9802,1994$.

[28] G. P. Mikitik and E. H. Brandt, "Generation of a dc voltage by an ac magnetic field in type-II superconductors," Phys. Rev. B, vol. 64, no. 9, p. 092502, 2001

[29] C. Plummer and J. Evetts, "Dependence of the shape of the resistive transition on composite inhomogeneity in multifilamentary wires," IEEE Trans. Magnetics, vol. 23, no. 2, pp. 1179-1182, 1987.

[30] J. Rhyner, "Magnetic properties and ac-losses of superconductors with power law current voltage characteristics," Physica C, vol. 212, no. 3-4, pp. 292-300, 1993.

[31] S. C. Wimbush and N. M. Strickland, "A public database of high- temperature superconductor critical current data," IEEE Trans. Appl. Supercond. vol. 27, no. 4, 2016.

[32] N. Strickland, C. Hoffmann, and S. Wimbush, "A 1 ka-class cryogen- free critical current characterization system for superconducting coated conductors," Rev. Sci. Instr., vol. 85, no. 11, p. 113907, 2014

[33] V. M. Zermeño, K. Habelok, M. Stepién and F. Grilli, "A parameter-free method to extract the superconductors $\mathrm{j}(\mathrm{b}, \vartheta)$ field-dependence from infield current-voltage characteristics of high temperature superconductor tapes," Supercond. Sci. Technol., vol. 30, no. 3, p. 034001, 2017.

[34] D. Hu, M. D. Ainslie, J. Rush, J. H. Durrell, J. Zou, M. Raine, and D. Hampshire, "Dc characterization and $3 \mathrm{~d}$ modelling of a triangular, epoxyimpregnated high temperature superconducting coil," Supercond. Sci. Technol., vol. 28, no. 6, p. 065011, 2015.

[35] D. Hu, M. D. Ainslie, M. J. Raine, D. P. Hampshire, and J. Zou, "Modeling and comparison of in-field critical current density anisotropy in hightemperature superconducting (HTS) coated conductors," IEEE Trans. Appl. Supercond., vol. 26, no. 3,2016.

[36] Sundaram, A., Zhang, Y., Knoll, A.R., Abraimov, D., Brownsey, P., Kasahara, M., Carota, G.M., Nakasaki, R., Cameron, J.B., Schwab, G. and Hope, L.V., "2G HTS wires made on $30 \mu \mathrm{m}$ thick Hastelloy substrate.", Supercond. Sci. Technol., 29(10), p.104007, 2016.

[37] Z. Jiang, R. Badcock, N. Long, M. Staines, K. Thakur, L. Lakshmi, Wright, K. Hamilton, G. Sidorov, R. Buckley et al., "Transport ac loss characteristics of a nine strand ybco roebel cable," Supercond. Sci. Technol, vol. 23, no. 2, p. 025028,2010 